University of Wollongong

Research Online

Faculty of Engineering and Information

Faculty of Engineering and Information

Sciences - Papers: Part A

Sciences

$1-1-2012$

\title{
Pedestrian lane detection for the visually impaired
}

Manh Cuong Le

University of Wollongong

Son Lam Phung

University of Wollongong, phung@uow.edu.au

Abdesselam Bouzerdoum

University of Wollongong, bouzer@uow.edu.au

Follow this and additional works at: https://ro.uow.edu.au/eispapers

Part of the Engineering Commons, and the Science and Technology Studies Commons

Research Online is the open access institutional repository for the University of Wollongong. For further information contact the UOW Library: research-pubs@uow.edu.au 


\title{
Pedestrian lane detection for the visually impaired
}

\begin{abstract}
Traveling safely in outdoor environments is one of the most challenging activities for vision-disabled people. To improve the mobility of these people, an assistive navigation system is necessary. This paper is a step towards developing this system. We propose a robust method to detect the pedestrian marked lane at traffic junctions. The proposed method involves three stages: patches of interest (POI) extraction, lane marker detection and lane detection. The POI extraction is first performed to detect image patches located on the lane marker boundaries using template matching. Potential lane markers are then formed by using shape and intensity information. Finally, the walkable lane region is determined by verifying pairs of detected potential makers. A probabilistic framework using multiple geometric cues is proposed for the verification step. We create a new dataset collected from diverse environments and evaluate the proposed method on this dataset. The experimental results under challenging conditions have illustrated the efficiency and robustness of the proposed method compared to conventional techniques. (C) 2012 IEEE.
\end{abstract}

\section{Keywords}

impaired, pedestrian, lane, detection, visually

Disciplines

Engineering | Science and Technology Studies

\section{Publication Details}

M. Cuong. Le, S. Phung \& A. Bouzerdoum, "Pedestrian lane detection for the visually impaired," in 2012 International Conference on Digital Image Computing Techniques and Applications, DICTA 2012, 2012, pp.6. 


\title{
Pedestrian Lane Detection For the Visually Impaired
}

\author{
Manh Cuong Le, Son Lam Phung and Abdesselam Bouzerdoum \\ School of Electrical, Computer and Telecommunications Engineering, \\ University of Wollongong, Australia
}

\begin{abstract}
Traveling safely in outdoor environments is one of the most challenging activities for vision-disabled people. To improve the mobility of these people, an assistive navigation system is necessary. This paper is a step towards developing this system. We propose a robust method to detect the pedestrian marked lane at traffic junctions. The proposed method involves three stages: patches of interest (POI) extraction, lane marker detection and lane detection. The POI extraction is first performed to detect image patches located on the lane marker boundaries using template matching. Potential lane markers are then formed by using shape and intensity information. Finally, the walkable lane region is determined by verifying pairs of detected potential makers. A probabilistic framework using multiple geometric cues is proposed for the verification step. We create a new dataset collected from diverse environments and evaluate the proposed method on this dataset. The experimental results under challenging conditions have illustrated the efficiency and robustness of the proposed method compared to conventional techniques.
\end{abstract}

\section{INTRODUCTION}

A significant percentage of the world population suffers from visual impairment and blindness. According to the World Health Organization, globally about 285 million people are visually impaired, and 39 million of them are blind [1]. The most difficulty of the visually impaired in everyday activities is inability to travel safely and independently [2]. To cope with this problem, an assistive navigation system for outdoor environments is necessary. The system should perform several vital micro-navigation tasks such as finding pedestrian lanes, detecting and recognising traffic obstacles, and sensing dangerous traffic situations.

Conventional travel aids for blind people include white canes and guide dogs. A white cane assists blind people in detecting obstacles and the walking paths at a close range. Compared with the white cane, a guide dog is more versatile in evading obstacles and hazards, and following a familiar route, but it has a high cost and only assists blind people in the familiar environment. To improve the mobility of blind people, technology aids have been developed. A popular technology aid of navigation for the blind is obstacle detection systems, e.g the GuideCane [3] and the Miniguide [4]. Furthermore, GPS-based systems [5], [6] have been developed to support the blind in finding routes and locations. Other assistive systems of embedded infrastructures, e.g Talking Signs [7] and Audible Pedestrian Signals [8], allow the travelers to find and recognise landmarks, signs and facilities of interest, and to know when to cross intersections. However, pedestrian lane detection for the visually impaired is still absent in the above systems.
This paper addresses the computer vision task of detecting pedestrian lanes that are located by painted markers. Although lane detection for autonomous vehicles has been investigated for several decades [9]-[11], little work has been done on pedestrian lane detection for blind people [12], [13]. Furthermore, most techniques for detecting pedestrian lanes are designed to find zebra painted patterns [12]-[14].

In this paper, a new method is proposed to detect pedestrian lanes that are marked by two white stripes; these are common cases at traffic intersections (see Fig. 1). Our method includes three stages: patches of interest extraction, lane marker detection and lane detection. Conventionally, the Hough transform technique for line detection is often applied to detect the boundaries of lane markers [15]-[17]. However, this approach is sensitive to cluttered backgrounds which affect the Hough map. In our method, the boundaries of lane markers are not extracted using edge features. Instead, appearance information of local regions on the maker boundaries is used. Image patches with their centres located on or close to the lane marker boundaries (see Fig. 2) are considered as patches of interest. POI extraction is first performed by using normalised cross-correlation template matching. Once POI have been obtained, potential lane markers are detected by grouping POI using shape and intensity features. The walkable lane region is finally detected by verifying pairs of these potential markers. A probabilistic framework is proposed for this verification step using geometric cues of lane marker pairs.

In comparison with using edge pixels, using the image patches for extracting the marker boundaries has several advantages. First, the image patches are more informative and discriminative. Second, matching image patches is less sensitive to illumination changes. Third, since the image patches are not located on edge pixels, their detection is not affected by the performance of edge detectors.

Most existing work on marked lane detection is evaluated based on visual inspection. We address this gap by creating a large dataset of images with the ground-truth. This allows the performance of algorithms for pedestrian lane detection to be evaluated rigorously. The proposed method is evaluated and compared with existing methods on this newly created dataset.

This paper is organized as follows. We briefly review related works in Section II. The proposed method is then described in Section III. Experimental results and comparisons are presented in Section IV. The conclusion is drawn in Section V. 


\section{RELATED WORK}

Since most interests in marked lane detection have been for vehicle navigation, we review the existing methods and their suitability to detecting pedestrian marked lanes.

Existing lane detection methods can be categorised as edge-based methods [12], [15]-[17] or intensity/colour-based methods [9], [10]. Edge-based methods often extract edges in the image and then use line detection algorithms, e.g. the Hough transform, to obtain the lane marker boundaries. The detected lines are then verified with a predefined lane model (e.g. straight lines, B-Splines, parabola, or hyperbola) to identify the true lane markers. The edge-based approach is simple in implementation, but it also has certain drawbacks. First, its performance depends on the edge detectors used. Most existing edge detectors are affected by edge thresholds, e.g. too high or too low thresholds used in the Canny detector [18]. A low threshold will include a large number of unwanted pixels from the cluttered background, while a high threshold will omit important edge pixels. Second, detecting lane markers using the Hough transform is sensitive to scenes that include many extraneous edges. In assistive navigation for blind people, cluttered backgrounds are often obtained (see Fig. 1). Therefore, using edges and line detection methods (e.g. the Hough transform) for detecting lane marker boundaries would have a low speed and accuracy.

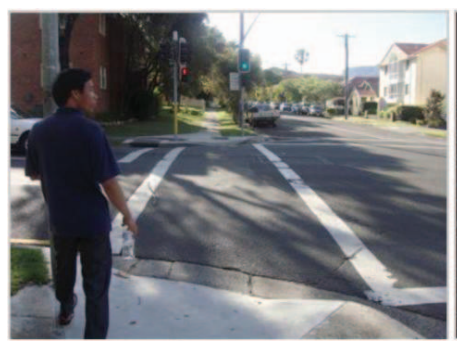

(a)

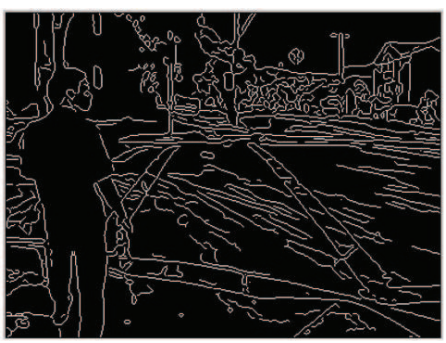

(b)
Fig. 1. Example of edge detection: (a) original image, (b) edge map extracted by Canny detector.

Alternatively, intensity or colour information is employed to segment lane markers [9]-[11], [19]. Based on the observation that lane markers have higher intensity than surrounding road areas, intensity patterns of lane markers are used. Examples of these intensity patterns include intensity-bump (darkbright-dark) filters [19], [20], steerable filters [21] and local thresholding [9]. Other methods exploit colour information for segmenting lane marker regions [10], [22], [23]. However, these techniques are affected by weather and illumination conditions. In severe environment conditions, e.g. extreme illumination changes or dark scenes, there is little difference in colour and intensity between lane markers and road surface. In addition, unwanted regions such as the sky and cars (with white colour) do not differ from lane markers using only intensity or colour.

Another drawback of existing vehicle lane detection methods is that they require the prior knowledge such as camera's parameters [11], [24] and scene's layout [25], [26]. However, this information is often not available in assistive navigation systems for blind people.

\section{PROPOSED METHOD}

The proposed method includes three stages: POI extraction, lane marker detection, and lane detection. In the following, we describe each stage in details.

\section{A. Patches of interest extraction}

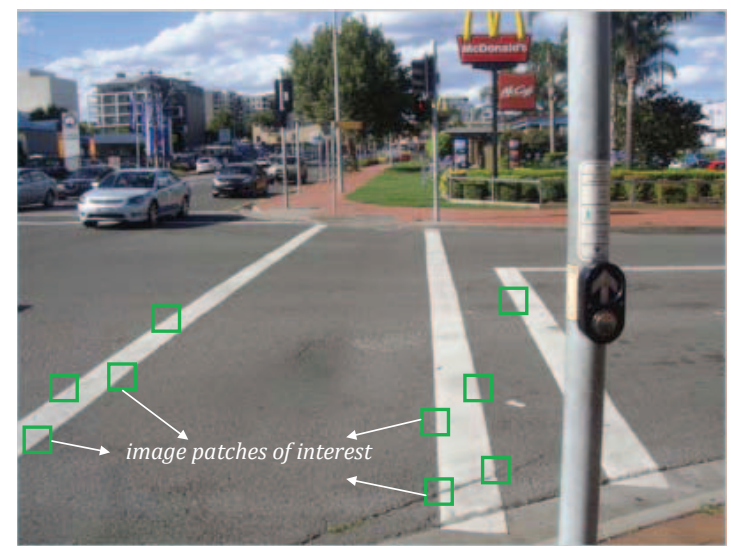

Fig. 2. Example image patches of interest.

We propose a method to extract local regions on the boundaries of lane markers (see Fig. 2). These regions are considered as POI. In comparison with the inner regions of lane markers, the POI include local appearance of both lane markers and road surface, and therefore provide a more informative and discriminative power. In contrast, the inner regions of lane markers often contain uniform patterns of intensity or colour, and therefore are not discriminative from several non-marker regions (e.g. the sky). The POI also implicitly include edge information, but they do not depend on edge detectors. POI extraction can be performed by using complex classifiers such as support vector machine (SVM). However, this method is computationally intensive and not suitable for real-time detection [11]. In our method, the POI are extracted using normalised cross-correlation (NCC) template matching. An advantage of NCC template matching is that it is less sensitive to illumination changes.

The templates are created as follows. Consider a set of training images with lane markers that are annotated manually. For each point on the lane marker boundaries, an image patch of size $(2 W+1) \times(2 W+1)$ centered at that point is extracted. All these image patches are then grouped into $N$ clusters using the $K$-means algorithm. The centres of the clusters are considered as templates and stored in a codebook $C$.

The similarity between two patches $p$ and $q$ is defined by the normalized cross-correlation measure $\operatorname{NCC}(p, q)$ as follows:

$$
\operatorname{NCC}(p, q)=\frac{\sum_{i}\left(q_{i}-\bar{q}\right)\left(p_{i}-\bar{p}\right)}{\sqrt{\sum_{i}\left(q_{i}-\bar{q}\right)^{2} \sum_{i}\left(p_{i}-\bar{p}\right)^{2}}},
$$




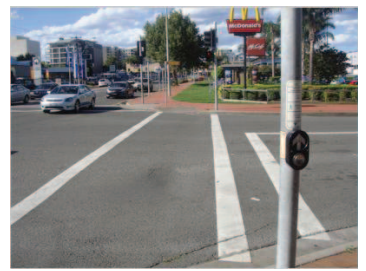

(a)

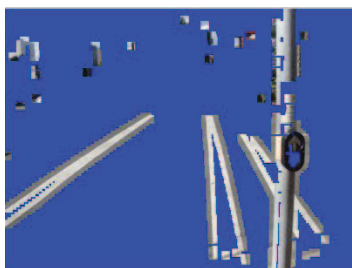

(b)

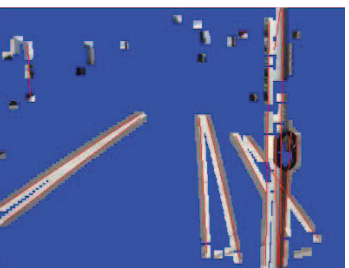

(c)

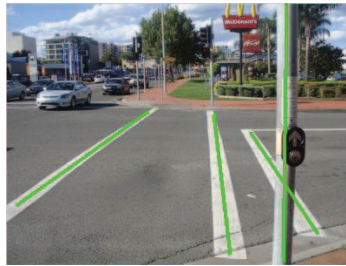

(d)

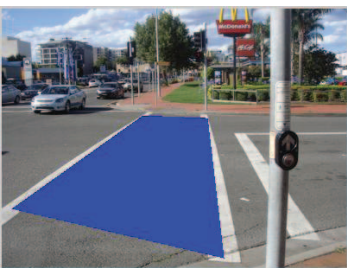

(e)

Fig. 3. Illustration of the proposed method: (a) input image, (b) result of POI extraction, (c) result of line detection, (d) result of lane marker detection (note that each lane marker is represented by its central line), and (e) result of lane detection.

where $p_{i}$ and $q_{i}$ is the colour values of the $i$-th pixel of $p$ and $q$, and $\bar{p}$ and $\bar{q}$ are the mean colour values of $p$ and $q$.

For a test image, POI extraction is performed as follows. We scan the input image by a local window of size $(2 W+1) \times(2 W+1)$ with the horizontal and vertical stride steps of $\left(d_{x}, d_{y}\right)$. For each image patch located on local windows, its best matching template in the codebook $C$ is determined by the maximum NCC value. If this $\mathrm{NCC}$ value is lower than a threshold $T_{P}$, the image patch is not considered as a patch of interest, and is therefore discarded. The value $W$ is selected so that the image patches contain sufficient information of the marker and road surface patterns. Too small or too large $W$ will affect the accuracy and computation complexity of POI extraction. Similarly, the threshold $T_{P}$ needs to be tunned. If this threshold is set either too low or too high, a large number of background image patches will be included or POI may be omitted. For stride steps $\left(d_{x}, d_{y}\right)$, if their values are too small, the computation complexity of POI extraction is high. If $\left(d_{x}, d_{y}\right)$ values are too large, the number of extracted POI will be small and therefore affects the accuracy of lane marker detection.

Figure $3 \mathrm{~b}$ illustrates an example result of POI extraction. As can be seen, the background image patches are still detected and they will be removed next.

\section{B. Lane marker detection}

In this step, potential lane markers are detected from the extracted POI. The markers of pedestrian lanes are straight stripes, and this is a significant feature for finding potential borders of lane markers and removing the background image patches. In our work, marker borders are first detected using the random sample consensus (RANSAC) technique. Potential lane markers are then determined from the detected borders, using shape and intensity cues.

After the image patches are extracted, their centres are determined. Note that these centres are not necessarily the edge pixels of the markers. We apply the RANSAC technique on the set of these centres to detect straight lines. This stage results in a set of candidate marker borders. The RANSAC method is selected instead of the Hough transform because it is more tolerant to the marker boundaries that are not fully straight. We will compare the robustness of the RANSAC method and the Hough transform in Section IV. Figure 3c shows an example result of marker border detection.
Because of the perspective projection of images and the results of the RANSAC method, the vertical borders (along with the $y$-axis) of lane markers are more prominent compared with the horizontal borders. Therefore, in this step, we only use the vertical borders to determine the markers.

A true lane marker often has an elongated shape and high contrast compared with its surrounding regions, and this is important cues to determine a candidate lane marker from the detected borders.

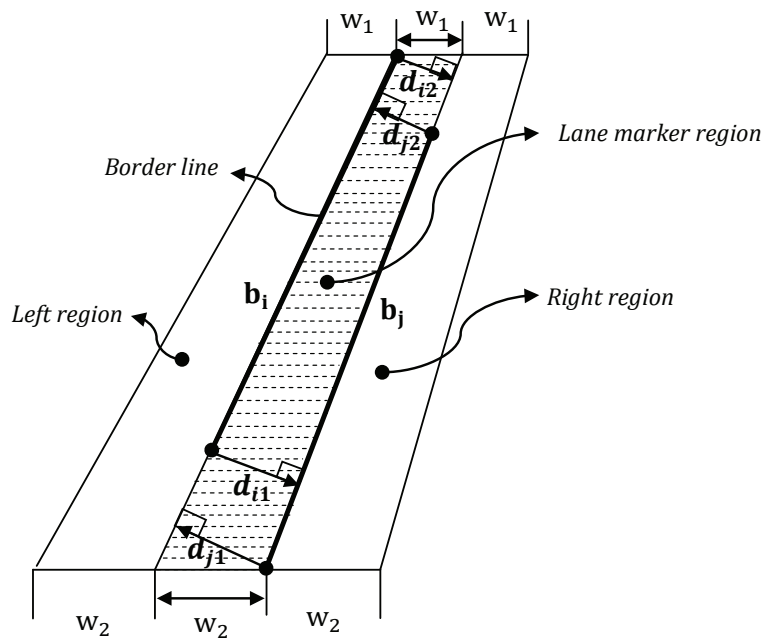

Fig. 4. Geometric relations between two borders of a lane marker (shaded area), and relations of a lane marker and its neighbouring regions.

For a border pair $\left(b_{i}, b_{j}\right)$, the elongation of the marker shape is measured as

$$
r_{i j}=\frac{d_{i 1}+d_{i 2}+d_{j 1}+d_{j 2}}{l_{i}+l_{j}}
$$

where $d_{i 1}$ and $d_{i 2}$ are distances from the end points of $b_{i}$ to $b_{j}$, $d_{j 1}$ and $d_{j 2}$ are distances from the end points of $b_{j}$ to $b_{i}$ (see Fig. 4), and $l_{i}$ and $l_{j}$ are the length of $b_{i}$ and $b_{j}$ respectively. Equation (2) indicates that the longer is the marker, the smaller is $r_{i j}$.

The intensity pattern of the marker formed by $\left(b_{i}, b_{j}\right)$ is represented by the ratio:

$$
g_{i j}=\frac{\bar{I}_{i j}}{\max \left(\bar{I}_{i j}^{\text {left }}, \bar{I}_{i j}^{\text {right }}\right)}
$$


where $\bar{I}_{i j}$ is the mean value of intensity of all pixels on the image region determined by $\left(b_{i}, b_{j}\right)$ (see the shaded area in Fig. 4), $\bar{I}_{i j}^{\text {left }}$ and $\bar{I}_{i j}^{\text {right }}$ are the mean values of intensities of the left and right regions as shown in Fig. 4. Equation (3) means that if the higher is the intensity contrast of a lane marker, the higher is $g_{i j}$.

A pair of detected lines $\left(b_{i}, b_{j}\right)$ is considered as the potential borders of a lane marker if the following conditions are satisfied:

$$
\left\{\begin{array}{l}
r_{i j} \leq T_{r} \\
g_{i j} \geq T_{g}
\end{array}\right.
$$

where $T_{r}$ and $T_{g}$ are predefined thresholds. The conditions in (4) mean that a true marker should have an elongated shape and high contrast of intensity compared with its surrounding regions. To determine the values of $T_{r}$ and $T_{g}$, the distributions for intensity patterns and shape elongations of the lane markers are first estimated from the training set, and then the threshold values are found based on these distributions.

This step results in a set of candidate lane markers. For each candidate lane marker, the central line is computed from its borders. An example result of lane marker detection is shown in Fig. 3d.

\section{Lane detection}

In this section, a probabilistic method is proposed to identify a pair of lane markers which best represents a true pedestrian lane region. This method is based on the geometric relations of two lane markers. For a pair of lane markers $\left(m_{i}, m_{j}\right)$, the geometric relations include the angle $\varphi_{i j}$ and the vertical overlap $o_{i j}$ between $m_{i}$ and $m_{j}$, as shown in Fig. 5. The conditional probability of the lane region $\mathcal{L}$ for a pair of lane markers $\left(m_{i}, m_{j}\right)$ is computed as

$$
\begin{aligned}
P\left(\mathcal{L} \mid m_{i}, m_{j}\right) & =\frac{p\left(m_{i}, m_{j} \mid \mathcal{L}\right) P(\mathcal{L})}{p\left(m_{i}, m_{j}\right)} \\
& =\frac{p\left(\varphi_{i j}, o_{i j} \mid \mathcal{L}\right) P(\mathcal{L})}{p\left(m_{i}, m_{j}\right)} .
\end{aligned}
$$

We assume that $\varphi_{i j}$ and $o_{i j}$ are statistically independent for a given $\mathcal{L}, p\left(m_{i}, m_{j}\right)$ is uniform, and $P(\mathcal{L})$ is constant. Equation (5) is written as

$$
P\left(\mathcal{L} \mid m_{i}, m_{j}\right) \propto p\left(\varphi_{i j} \mid \mathcal{L}\right) p\left(o_{i j} \mid \mathcal{L}\right),
$$

where $p\left(\varphi_{i j} \mid \mathcal{L}\right)$ and $p\left(o_{i j} \mid \mathcal{L}\right)$ represent the likelihood functions of geometric parameters for pairs of lane markers.

The $p\left(\varphi_{i j} \mid \mathcal{L}\right)$ is computed from the probability distribution function $(p d f)$ of the angle $\varphi_{i j}$ between the central lines of two markers of the true lanes given in the training dataset. The $p d f$ can be estimated by using various approaches such as Parzen window and Gaussian mixture. In this work, we use the histogram approach. The angles $\varphi_{i j}$ within the range $\left[0^{0}, 180^{\circ}\right]$ are quantised into $K$ bins.

The vertical overlap $o_{i j}$ between $m_{i}$ and $m_{j}$ is defined as

$$
o_{i j}=\frac{\min \left(m_{i}^{b}, m_{j}^{b}\right)-\max \left(m_{i}^{t}, m_{j}^{t}\right)}{\max \left(m_{i}^{b}, m_{j}^{b}\right)-\min \left(m_{i}^{t}, m_{j}^{t}\right)},
$$

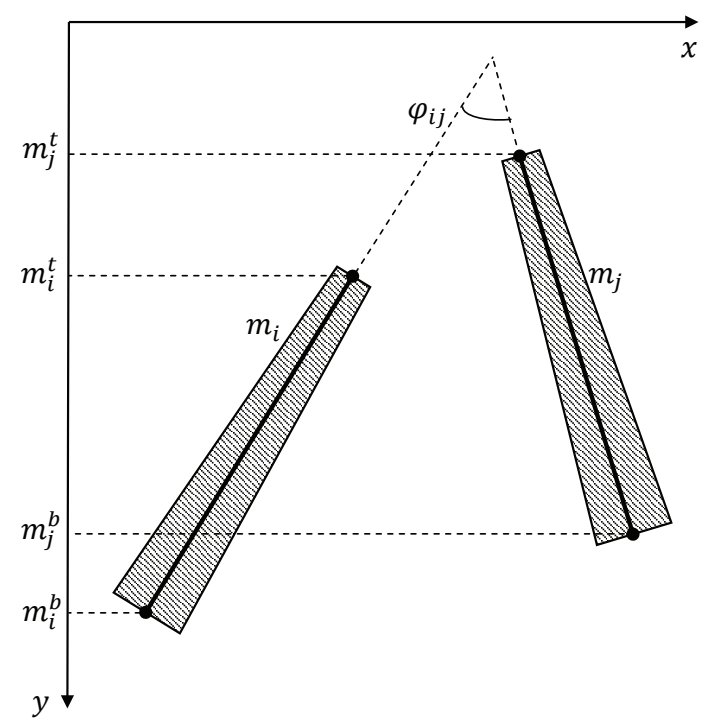

Fig. 5. Geometric relationship of two lane markers (shaded areas).

where $m^{t}$ and $m^{b}$ are the top and bottom coordinates of the central line of $m$ respectively (see Fig 5). Note that the image coordinate is employed, i.e. $(0,0)$ is the left-top corner of the image. Due to the perspective projection, the markers of a pedestrian lane have a high vertical overlap (see Fig 5). Thus, we model $p\left(o_{i j} \mid \mathcal{L}\right)$ for the vertical overlap of the detected lane markers, using the sigmoid function. The $p\left(o_{i j} \mid \mathcal{L}\right)$ is modelled as

$$
p\left(o_{i j} \mid \mathcal{L}\right)=\frac{\alpha}{\alpha+e^{-\beta o_{i j}}},
$$

where $\alpha$ and $\beta$ are positive parameters to control the vertical deviation of lane markers. Equation (8) indicates that the higher is the vertical overlap, the higher is $p\left(o_{i j} \mid \mathcal{L}\right)$.

Finally, the best candidate markers $m_{i}^{*}, m_{j}^{*}$ are obtained from the given set of candidate lane markers as

$$
\left(m_{i}^{*}, m_{j}^{*}\right)=\underset{m_{i}, m_{j}}{\arg \max } P\left(\mathcal{L} \mid m_{i}, m_{j}\right) .
$$

An example result of lane detection is illustrated in Fig. 3e.

\section{EXPERIMENTAL RESULTS}

This section presents the image data, evaluation measures and the results of several methods for pedestrian lane detection.

\section{A. Image Data and Evaluation Measures}

To evaluate algorithms for pedestrian lane detection, we acquired a large dataset of images, taken at traffic crossings with different backgrounds, times of day, and weather conditions. We created the ground-truth by manually annotating the image coordinates of pedestrian lane markers. Each lane marker was represented by a polygon. Lane regions then are obtained from the annotated markers. Overall, 1000 images of different scenes were collected. We used 600 images for training and the remaining images for testing. 
Given a ground-truth lane region $\mathbf{R}_{g}$ and a detected lane region $\mathbf{R}_{d}$, the matching score between these two regions is defined as

$$
s\left(\mathbf{R}_{g}, \mathbf{R}_{d}\right)=\frac{\left|\mathbf{R}_{g} \cap \mathbf{R}_{d}\right|}{\left|\mathbf{R}_{g} \cup \mathbf{R}_{d}\right|},
$$

where $\cap$ and $\cup$ denote the intersection and union of two regions, and $|\mathbf{R}|$ is the area of region $\mathbf{R}$.

A detected lane region $\mathbf{R}_{d}$ is considered as correct if there exists a ground-truth lane region $\mathbf{R}_{g}$ that satisfies

$$
s\left(\mathbf{R}_{g}, \mathbf{R}_{d}\right) \geq \theta,
$$

where $\theta$ is an evaluation threshold.

To evaluate the performance of methods for pedestrian lane detection, we use two measures:

- Recall is the percentage of the ground-truth lane regions that are detected correctly by machine.

- Precision is the ratio between the number of the lane regions detected correctly and the total number of machinegenerated lane regions.

\section{B. Results}

In our experiments, the parameters and threshold values are chosen as follows. The size of POI, horizontal and vertical stripe steps, the threshold value and the number of clusters in Section III-A are set as $W=3,\left(d_{x}, d_{y}\right)=(1,3), T_{P}=0.9$ and $N=150$. The threshold values $T_{r}$ and $T_{g}$ in (4) estimated automatically are 0.3 and 1.2 respectively. The parameters, evaluation threshold in (8) and (11), and the number of orientation bins in Section III-C are chosen as $\alpha=0.2, \beta=5.0, \theta=0.5$ and $K=18$.

We implemented the proposed method using Matlab and evaluated it on the newly created dataset. Sample visual results of pedestrian lane detection are presented in Fig. 6. By using appearance information of local regions on the marker boundaries, the proposed method detects robustly pedestrian lanes in different illumination and weather conditions (sunny, cloudy, strong shadows and times of day).

For comparison purposes, the following experiments were conducted:

- Using the Hough transform (HT) on the edge map, generated by the Canny detector, in detecting the borders of lane markers. This method is referred to as Edge+HT. The motivation of this experiment is to analyse the robustness of using POI and using edges.

- Applying the Hough transform on the extracted POI in estimating the borders of lane markers. This method is referred to as POI+HT. This experiment is used to investigate the effectiveness of using the RANSAC technique and the Hough transform.

Experimental results are summarised in Table I. Using POI for locating the borders of lane markers significantly outperforms the conventional approach of using edge pixels. This is because the POI extraction removes a large number of unwanted pixels. Through experiments, we have found that the POI+HT method achieved a detection rate (recall) of $91.0 \%$

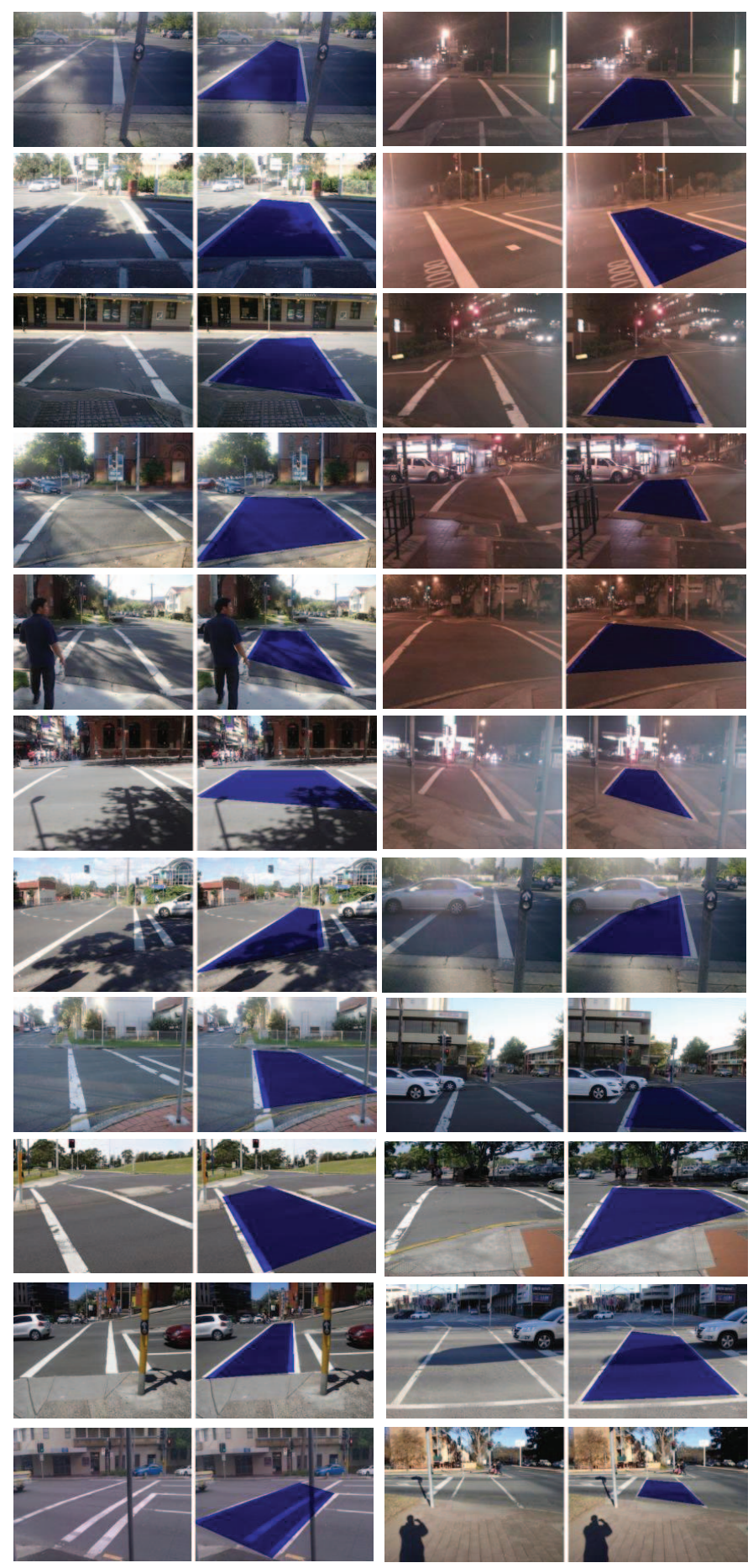

Fig. 6. Visual results of lane detection. Columns 1 and 3: input images. Columns 2 and 4: detected pedestrian lane regions.

and a precision of $94.0 \%$, whereas the Edge+HT method obtained only a detection rate of $86.7 \%$ and a precision of $90.4 \%$.

Experimental results also show the robustness of the RANSAC algorithm in comparison with the Hough transform. The POI+RANSAC method detected correctly $93.4 \%$ of lane 
TABLE I

COMPARISON OF ALGORITHMS FOR PEDESTRIAN LANE DETECTION.

\begin{tabular}{|l|c|c|c|}
\hline Methods & $\begin{array}{c}\text { Recall } \\
(\%)\end{array}$ & $\begin{array}{c}\text { Precision } \\
(\%)\end{array}$ & $\begin{array}{c}\text { Processing } \\
\text { time }(\mathrm{s})\end{array}$ \\
\hline Edge+Hough transform & 86.7 & 90.4 & 1.5 \\
\hline POI+Hough transform & 91.0 & 94.0 & 1.3 \\
\hline POI+RANSAC (proposed) & 93.4 & 95.2 & 1.2 \\
\hline
\end{tabular}

regions with a precision of $95.2 \%$, while the POI+HT method could detect only $91.0 \%$ of lane regions with a precision of $94.0 \%$.

We also compared the proposed method with the Edge+HT and POI+HT methods in terms of computational complexity. To process a $300 \times 400$ image, our Matlab implementation for the proposed method and the POI+HT method took an average time of 1.2 and 1.3 seconds, respectively. In comparison, on the same dataset, the Edge+HT method incurred a processing time of 1.5 seconds per image. This again shows the role of POI in removing unwanted pixels, and this improves the speed of the detection method, especially for the scenes that contain many cluttered backgrounds. We have found that the processing time of the proposed method is sufficient for navigation of blind people, but it should be further optimized.

\section{CONCLUSION}

This paper presented a robust method for detecting pedestrian marked lanes at traffic crossings. The proposed method detects the boundaries pixels of lane markers using local appearance information of both lane markers and road surface. The candidate boundaries are then estimated by using the RANSAC algorithm. Based on the detected boundaries, lane markers are determined by using shape and intensity features, and verified by a probabilistic framework with multiple geometric cues. Experimental results have shown that the proposed method is able to robustly detect pedestrian marked lanes under challenging environmental conditions. Furthermore, this paper created a benchmarking database, and an evaluation framework for pedestrian lane detection.

\section{REFERENCES}

[1] WHO, "Visual impairment and blindness," Tech. Rep. Fact Sheet No. 282, 2011. [Online]. Available: http://www.who.int/mediacentre/factsheets/fs282/en/ 1

[2] P. Strumillo, "Electronic interfaces aiding the visually impaired in environmental access, mobility and navigation," in Conference on Human System Interactions, 2010, pp. 17-24. 1

[3] J. Borenstein and I. Ulrich, "The guidecane - a computerized travel aid for the active guidance of blind pedestrians," in IEEE International Conference on Robotics and Automation, vol. 2, 1997, pp. 1283-1288. 1

[4] GDP, "The miniguide ultrasonic mobility aid," Tech. Rep., 2011 [Online]. Available: http://www.gdp-research.com.au 1

[5] H. Petrie, V. Johnson, T. Strothotte, A. Raab, R. Michel, L. Reichert, and A. Schalt, "MoBIC: An aid to increase the independent mobility of blind travellers," British Journal of Visual Impairment, vol. 15, pp. 63-66, 1997. 1
[6] J. M. Loomis, R. L. Klatzky, and R. Golledge, "Navigating without vision: basic and applied research," Optometry and vision science, vol. 78, pp. 282-289, 2001. 1

[7] W. Crandall, B. Bentzen, L. Myers, and J. Brabyn, "New orientation and accessibility option for persons with visual impairment: transportation applications for remote infrared audible signage," Clinical and Experimental Optometry, vol. 84, pp. 120-131, 2001. 1

[8] J. M. Barlow, B. L. Bentzen, and B. T., "Blind pedestrians and the changing technology and geometry of signalized intersections: Safety, orientation, and independence," Journal of Visual Impairment and Blindness, vol. 99, pp. 587-598, 2005. 1

[9] P. Charbonnier, F. Diebolt, Y. Guillard, and F. Peyret, "Road markings recognition using image processing," in IEEE Conference on Intelligent Transportation System, 1997, pp. 912-917. 1, 2

[10] H. Cheng, B. Jeng, P. Tseng, and K. C. Fan, "Lane detection with moving vehicles in the traffic scenes," IEEE Transactions on Intelligent Transportation Systems, vol. 7, no. 4, pp. 571-582, 2006. 1, 2

[11] Z. W. Kim, "Robust lane detection and tracking in challenging scenarios," IEEE Transactions on Intelligent Transportation Systems, vol. 9, no. 1, pp. 16-26, 2008. 1, 2

[12] S. Se, "Zebra-crossing detection for the partially sighted," in IEEE Conference on Computer Vision and Pattern Recognition, 2000, pp. 211217. 1,2

[13] V. Ivanchenko, J. Coughlan, and S. Huiying, "Detecting and locating crosswalks using a camera phone," in IEEE Conference on Computer Vision and Pattern Recognition, 2008, pp. 1-8. 1

[14] M. S. Uddin and T. Shioyama, "Bipolarity and projective invariant-based zebra-crossing detection for the visually impaired," in IEEE Conference on Computer Vision and Pattern Recognition, 2005, pp. 22-30. 1

[15] Y. Wang, E. K. Teoh, and D. Shen, "Lane detection and tracking using b-snake," Image and Vision Computing, vol. 22, no. 4, pp. 269-280, 2004. 1, 2

[16] Q. Li, N. Zheng, and H. Cheng, "Springrobot: a prototype autonomous vehicle and its algorithms for lane detection," IEEE Transactions on Intelligent Transportation Systems, vol. 5, no. 4, pp. 300-308, 2004. 1 , 2

[17] D. Schreiber, B. Alefs, and M. Clabian, "Single camera lane detection and tracking," in IEEE Conference on Intelligent Transportation Systems, 2005, pp. 302-307. 1, 2

[18] J. Canny, "A computational approach to edge detection," IEEE Transaction on Pattern Analysis and Machine Intelligence, vol. 8, pp. 679-698, 1986. 2

[19] S.-S. Ieng, J.-P. Tarel, and R. Labayrade, "On the design of a single lane-markings detectors regardless the on-board camera's position," in IEEE Intelligent Vehicles Symposium, 2003, pp. 564-569. 2

[20] M. Bertozzi and A. Broggi, "Gold: a parallel real-time stereo vision system for generic obstacle and lane detection," IEEE Transactions on Image Processing, vol. 7, no. 1, pp. 62-81, 1998. 2

[21] J. C. McCall and M. M. Trivedi, "Video-based lane estimation and tracking for driver assistance: survey, system, and evaluation," IEEE Transactions on Intelligent Transportation Systems, vol. 7, no. 1, pp. 20-37, 2006. 2

[22] Y. He, H. Wang, and B. Zhang, "Color-based road detection in urban traffic scenes," IEEE Transactions on Intelligent Transportation Systems, vol. 5, no. 4, pp. 309-318, 2004. 2

[23] T. Y. Sun, S. Tsai, and V. Chan, "HSI color model based lane-marking detection," in IEEE Conference on Intelligent Transportation Systems, 2006, pp. $1168-1172.2$

[24] A. H. S. Lai and N. H. C. Yung, "Lane detection by orientation and length discrimination," IEEE Transactions on Systems, Man, and Cybernetics, Part B: Cybernetics, vol. 30, no. 4, pp. 539-548, 2000. 2

[25] K. Kaplan, C. Kurtul, and H. L. Akin, "Fast lane tracking for autonomous urban driving using hidden Markov models and multiresolution Hough transform," Industrial Robot: An International Journal, vol. 37, no. 3, 2010. 2

[26] T. Lei, Y.-Y. Fan, and L.-B. Huang, "Fast lane recognition based on morphological multi-structure element model," Optoelectronics Letters, vol. 5, no. 4, pp. 304-308, 2009. 2 